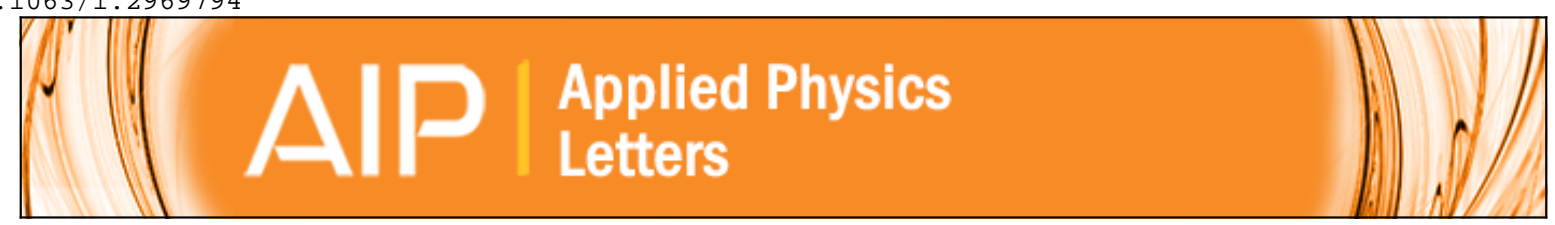

Thermal noise cancellation in symmetric magnetoelectric bimorph laminates Junyi Zhai, Zengping Xing, Shuxiang Dong, Jiefang Li, and D. Viehland

Citation: Applied Physics Letters 93, 072906 (2008); doi: 10.1063/1.2969794

View online: http://dx.doi.org/10.1063/1.2969794

View Table of Contents: http://scitation.aip.org/content/aip/journal/apl/93/7?ver=pdfcov

Published by the AIP Publishing

Over 700 papers \&
presentations on

multiphysics simulation www nows

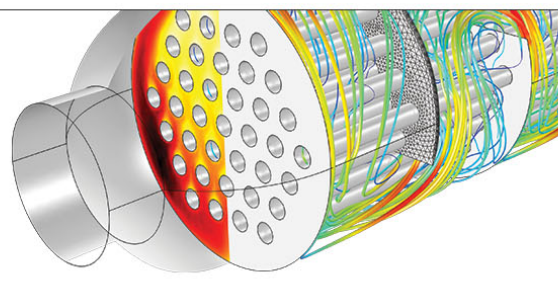




\title{
Thermal noise cancellation in symmetric magnetoelectric bimorph laminates
}

\author{
Junyi Zhai, ${ }^{a)}$ Zengping Xing, Shuxiang Dong, Jiefang Li, and D. Viehland \\ Department of Materials Science and Engineering, Virginia Tech, Blacksburg, Virginia 24061, USA
}

(Received 1 July 2008; accepted 23 July 2008; published online 22 August 2008)

\begin{abstract}
We have found a symmetric Terfenol-D/Pb(Zr,Ti)03 (PZT) bimorph magnetoelectric (ME) laminate, which operates in a bending mode under an unsymmetrical (U-shaped) magnetic bias. It has a giant ME voltage coefficient of about $70 \mathrm{~V} / \mathrm{cm}$ Oe at resonance. Unlike other symmetric ME laminate structures, the symmetric bimorph structure has the capability to reject thermal noise from a magnetic signal, due to its back-to-back structure. The mechanism for the thermal noise cancellation capability is that the laminate operates in a bending mode (out charges of reverse sign), whereas the thermal noise is contained in a longitudinal mode (out charges have the same sign, allowing cancellation by differential detection). (C) 2008 American Institute of Physics.
\end{abstract}

[DOI: $10.1063 / 1.2969794]$

The magnetoelectric (ME) effect has been widely investigated during the last ten years since giant $\mathrm{ME}$ effects were reported in piezoelectric/magnetostrictive laminate composites. ${ }^{1,2}$ The ME voltage coefficients found in Terfenol- $\mathrm{D} / \mathrm{Pb}\left(\mathrm{Mg}_{1 / 3} \mathrm{Nb}_{2 / 3}\right) \mathrm{O}_{3}-\mathrm{PbTiO}_{3}$ laminates ${ }^{3-5}$ are $>4 \mathrm{~V} / \mathrm{cm}$ Oe, which is $\sim 200 \times$ times larger than that of a single crystal $\mathrm{Cr}_{2} \mathrm{O}_{3}{ }^{6}{ }^{6}$ Recently, even larger ME coefficients have been found in Metglas-based ME laminates such as Metglas/polyvinylidene-flouride (PVDF) and Metglas/ PbZro.48Tio.5203 (PZT)-fiber composites. ${ }^{7,8}$ The ME effect in laminates is mainly dependent on several important parameters of the piezoelectric and magnetostrictive phases such as the piezoelectric voltage constant, piezomagnetic coefficient, elastic stiffness, dielectric permittivity, and magnetic permeability. ${ }^{9,10}$ Enhancements in the piezoelectric and/or magnetostriction tensor coefficient could assist in developing laminate composites with even larger $\mathrm{ME}$ coefficients.

In addition, the structure of ME laminates has an important impact on the ME effect. Four basic types of operational modes have been identified. ${ }^{11}$ These are the following. (i) the longitudinally magnetized and longitudinally poled or (L-L) mode, (ii) the longitudinal magnetized and transversely poled or (L-T) mode, (iii) the transversely magnetized and longitudinally poled or (T-L) mode, and (iv) the transversely magnetized and transversely poled or (T-T) mode. Even if Terfenol-D and PZT layers in these modes have the same dimensions, the ME coefficient of the four modes are significantly different. In general, the LL and TL modes have larger ME voltage coefficients than either the LT or TT ones. Furthermore, the LL and LT modes require smaller dc magnetic biases, relative to the TL and TT ones. This is because the demagnetization fields of the magnetostrictive layers in the transverse direction (T-T mode) are much larger than that in the longitudinal one. A unimorph type ME laminate is also known. ${ }^{12}$ It has an asymmetrical structure. Thus its first resonance mode is a bending one, rather than a longitudinal one as for the four basic laminate modes. By using an asymmetri-

${ }^{a)}$ Electronic mail: zhjy@vt.edu. cal structure operated in a bending mode, it is easy to decrease the first resonance frequency.

Giant ME effects in laminates have been investigated for highly sensitive, low frequency magnetic field sensors. ${ }^{13-15}$ However, as a magnetic sensor, the minimization in intrinsic ${ }^{16,17}$ and extrinsic noises is critical to achieving high sensitivity. In the case of ME sensors, the dominate noise sources are the following. (i) thermal noise, introduced via pyroelectric currents; and (ii) vibration or acoustic noises, introduced via the piezoelectric effect. Accordingly, small temperature fluctuations (or environment air flow and vibrations) will act as noise sources in the piezoelectric layer. Unfortunately, the four basic types of modes and unimorph structure, according to previous reports, do not have the capability to reject thermal noise. In this letter, we focus on thermal noise cancellation. A different ME laminate structure - the symmetric bimorph configuration operating in bending mode-is proposed, which has the ability to reject thermal noise from the detected magnetic signals.

Figure 1 shows the symmetric configuration of our bimorph laminate. It consists of two PZT layers and two Terfenol-D layers, bonded together by a hard epoxy. The PZT layers were poled in the thickness direction, where the polarization directions of the two layers were reversed with respect to each other. Both Terfenol-D layers were magnetized along their longitudinal directions; however, unlike prior modes, the magnetization directions in the two Terfenol-D layers were reversed by using two U-shaped dc magnetic biases. The dimensions of each Terfenol-D layer were $14 \times 6 \times 1.2 \mathrm{~mm}^{3}$. The dimensions of each PZT layer were $16 \times 6 \times 1 \mathrm{~mm}^{3}$. To compare the ME voltage coefficient of this symmetric bimorph mode to that of the LT one,

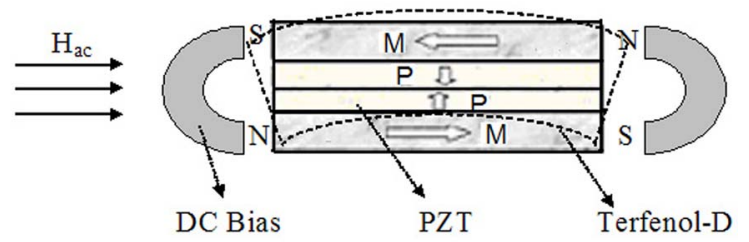

FIG. 1. (Color online) Schematic of the symmetric bimorph mode laminate with $\mathrm{U}$ shaped dc magnetic biases. 


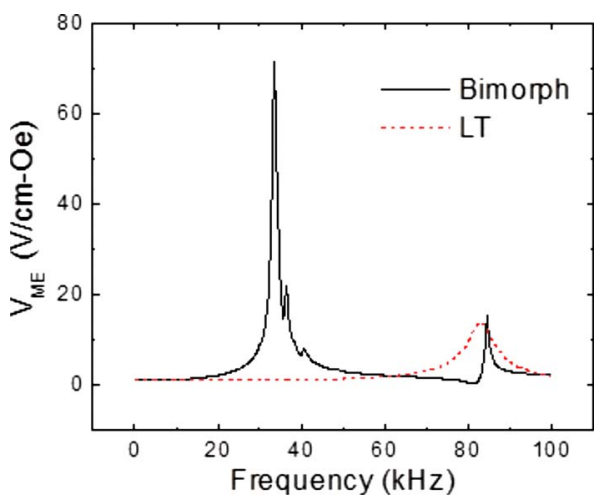

FIG. 2. (Color online) Frequency dependence $\left(10^{2}<f<10^{5} \mathrm{~Hz}\right)$ of the ME voltage coefficient for the symmetric bimorph and LT modes. These laminates have the same dimensions and where constructed from Terfenol-D and PZT layers of the same size.

a Terfenol-D/PZT four layer LT laminate of the same dimensions was constructed, where the polarization directions of both PZT layers were the same.

When an ac magnetic field $\left(H_{\mathrm{ac}}\right)$ is applied along the longitudinal direction, the magnetization $(M)$ in one Terfenol-D will decrease, whereas that in the other will increase: which will result in a decrease in the magnetostriction in one layer and an increase in the other. Thus, our symmetric bimorph laminate operates in a "pure" bending mode as a magnetic sensor, where the top and bottom PZT layers have induced charges of opposite signs. Thus, the differential output of these two PZT layers will be doubled, with respect to a single layer. Furthermore, when the temperature is changed, each layer in this symmetric structure will elongate or shrink together in a longitudinal mode. In this case, the top and bottom PZT layers will have induced thermal charges of the same sign: and consequently, the differential output of the thermal induced charge from the ME laminate will be essentially null. Theoretically, our ME symmetric bimorph should produce a high ME voltage, while at the same time rejecting thermal noise.

First, we measured the ME voltage coefficients of both the symmetric bimorph and a LT mode laminate. Figure 2 shows the measured frequency dependence of their ME voltage coefficients. In the quasistatic frequency range, the ME voltage coefficient of the symmetry bimorph and the LT mode were nearly equal $(\sim 1.2 \mathrm{~V} / \mathrm{cm}$ Oe). However, under resonance drive conditions, the ME coefficients of these two types of laminates were much different. The first resonance frequency of the symmetric bimorph was low $(\sim 34 \mathrm{kHz})$, whereas that of the LT mode was much higher $(\sim 83 \mathrm{kHz})$. In addition, a large ME voltage coefficient of $72 \mathrm{~V} / \mathrm{cm}$ Oe was found at the first bending mode resonance of the symmetric bimorph, which was much higher than that of the LT mode.

The lower resonance frequency of the symmetric bimorph is due to it being a bending mode. The bending mode resonance frequency of a laminate can be given as

$$
f_{n}^{B}=\frac{\pi d}{2 \sqrt{12} l^{2}} \frac{\left(n+\frac{1}{2}\right)^{2}}{\sqrt{\overline{\rho s}_{33}}}, \quad n=1,2,3, \ldots,
$$

where $f_{n}^{B}$ is the nth order bending resonance frequency, $d$ the total thickness of the laminate, $l$ is the length, $\bar{\rho}$ is the average density $\left(\bar{\rho}=v_{C} \rho_{T}+v_{P} \rho_{P}\right) \bar{s}_{33}$ is the equivalent

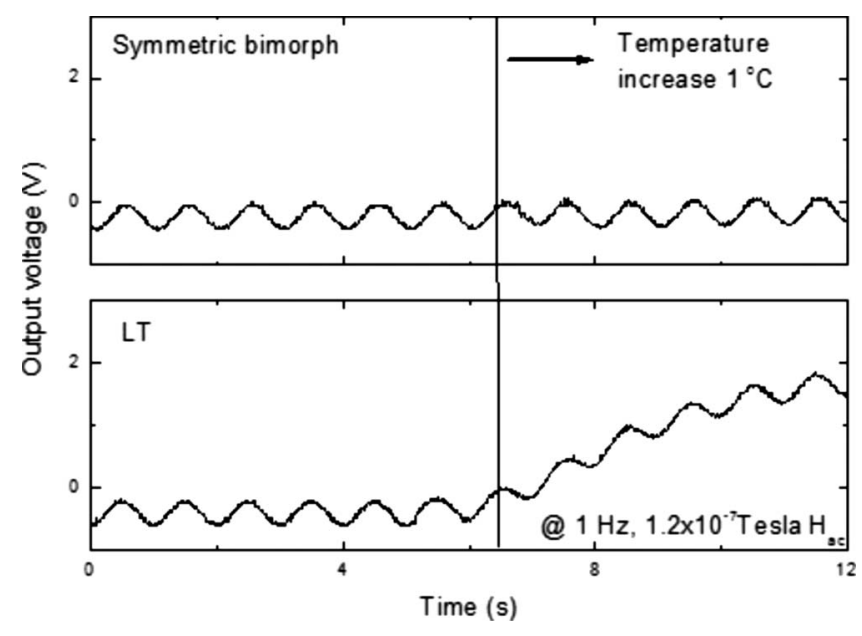

FIG. 3. Time dependence of the voltage induced by an ac magnetic field of $H_{\mathrm{ac}}=1.2 \times 10^{-7} \mathrm{~T}(f=1 \mathrm{~Hz})$ for (a) our symmetric bimorph, and (b) a LT mode laminate. At time $t=6.5 \mathrm{~s}$, temperature increased $1{ }^{\circ} \mathrm{C}$ by thermoelectric plate.

elastic compliance of the laminate $\left[\bar{s}_{33}=s_{33, T} s_{33, P} /\left(v_{P} s_{33, T}\right.\right.$ $\left.\left.+v_{T} s_{33, P}\right)\right], v$ is the volume fraction of Terfenol-D or PZT, and the subscripts $T$ and $P$ designate Terfenol-D and PZT respectively. Whereas, the nth order longitudinal mode resonance frequency $\left(f_{n}^{L}\right)$ is given by

$$
f_{n}^{L}=\frac{n}{2 l} \frac{1}{\sqrt{\overline{\rho s}_{33}}}, \quad n=1,, 3, \ldots .
$$

By using Eqs. (1) and (2), we calculated the first order bending and longitudinal mode frequencies. The theoretical predictions were in agreement with the experimentally observed first order resonance frequencies for the symmetric bimorph and LT modes, which were 34 and $83 \mathrm{kHz}$, respectively.

Next, we compared the effect of a thermal noise source on the ability of both the symmetric bimorph and LT laminates to sense small magnetic field changes in the time domain. The thermal noise source was generated by a thermoelectric plate which was controlled by a dc current amplifier. Figure 3 shows the time dependence of the ME voltage when subjected to a small $H_{\mathrm{ac}}$ of $1.2 \times 10^{-7} \mathrm{~T}$ at $1 \mathrm{~Hz}$. Data are shown for both types of laminates. When the temperature was kept constant, the symmetric bimorph and LT modes had similar output voltages, which had sinusoidal wave forms, corresponding to that of $H_{\mathrm{ac}}$. However, when the temperature was modulated by $1{ }^{\circ} \mathrm{C}$, the voltage signal of the LT mode laminate was offset to higher voltages, resulting in the accumulation of charge across the PZT layer via the pyroelectric effect. For symmetric bimorph, the same temperature flux did not result in any noticeable changes of the output voltage in the time domain. These results demonstrate the ability of the symmetric bimorph mode to reject a thermal noise source from a signal, due to its internal structure.

In summary, we have found a symmetric bimorph ME laminate that has the unique capability to reject thermal noise from a magnetic signal. It works at a bending mode, rather than longitudinal mode although it has a symmetric structure. At the first bending resonance, the ME voltage coefficient is as large as $70 \mathrm{~V} / \mathrm{cm} \mathrm{Oe}$, which is much higher than that of the LT mode. Our symmetric ME bimorph offers potential application as a magnetic sensor, due to its large ME properties and noise rejection capabilities. 
We gratefully acknowledge supported from the Office of Naval Research.

${ }^{1}$ M. Fiebig, J. Phys. D: Appl. Phys. 28, R123 (2005).

${ }^{2}$ C.-W. Nan, M. I. Bichurin, S. X. Dong, D. Viehland, and G. Srinivasan, J. Appl. Phys. 103, 031101 (2008).

${ }^{3}$ S. X. Dong, J.-F. Li, and D. Viehland, Appl. Phys. Lett. 83, 2265 (2003).

${ }^{4}$ S. X. Dong, J.-F. Li, and D. Viehland, Appl. Phys. Lett. 85, 5305 (2004).

${ }^{5}$ S. X. Dong, J. Y. Zhai, F. Bai, J.-F. Li, and D. Viehland, Appl. Phys. Lett. 87, 062502 (2005)

${ }^{6}$ G. T. Rado and V. J. Folen, Phys. Rev. Lett. 7, 310 (1961).

${ }^{7}$ J. Y. Zhai, S. X. Dong, Z. P. Xing, J.-F. Li, and D. Viehland, Appl. Phys. Lett. 89, 083507 (2006).

${ }^{8}$ S. X. Dong, J. Zhai, J.-F. Li, and D. Viehland, Appl. Phys. Lett. 89, 252904 (2006).
${ }^{9}$ C. W. Nan, Phys. Rev. B 50, 6082 (1994).

${ }^{10}$ G. Srinivasan, E. T. Rasmussen, J. Gallegos, R. Srinivasan, Y. I. Bokhan, and V. M. Laletin, Phys. Rev. B 64, 214408 (2001).

${ }^{11}$ S. X. Dong, J.-F. Li, and D. Viehland, IEEE Trans. Ultrason. Ferroelectr. Freq. Control 50, 1253 (2003).

${ }^{12}$ J. G. Wan, Z. Y. Li, Y. Wang, M. Zeng, and J.-M. Liu, Appl. Phys. Lett. 86, 202504 (2005).

${ }^{13}$ J. Y. Zhai, Z. P. Xing, S. Dong, J. F. Li, and D. Viehland, Appl. Phys. Lett. 88, 062510 (2006).

${ }^{14}$ S. X. Dong, J. Zhai, J.-F. Li, and D. Viehland, Appl. Phys. Lett. 88, 082907 (2006).

${ }^{15}$ J. Y. Zhai, S. Dong, Z. P. Xing, J. F. Li, and D. Viehland, Appl. Phys. Lett. 91, 123513 (2007)

${ }^{16}$ Z. P. Xing, J. F. Li, and D. Viehland, Appl. Phys. Lett. 91, 182902 (2007).

${ }^{17}$ Z. P. Xing, J. Y. Zhai, S. Dong, J. F. Li, and D. Viehland, Meas. Sci. Technol. 19, 015216 (2008). 